\title{
Giant urethral stone with diverticulum; a case report
}

Asad Ramzan ${ }^{a}$, Adeen Akram ${ }^{\text {b }}$, Suaiba Saif , Farhan Jamshed ${ }^{d}$, Nauman Khalid

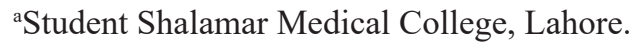

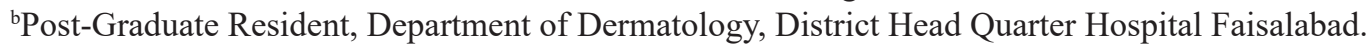

'Post-Graduate Resident, Department of Urology, Madinah Teaching Hospital Faisalabad.

${ }^{\mathrm{d} S e n i o r ~ R e g i s t r a r, ~ D e p a r t m e n t ~ o f ~ U r o l o g y ~ M a d i n a h ~ T e a c h i n g ~ H o s p i t a l ~ F a i s a l a b a d . ~}$

${ }^{e}$ Consultant, Department of Urology, Madinah Teaching Hospital Faisalabad.

*Corresponding author: Asadramzan61@yahoo.com

\section{ABSTRACT}

Urethral calculi are rare and represent $1-2 \%$ of all urinary stone diseases. Rarely, calculus grows to large size and are labeled as a "giant urethral calculus". A 75-year-old male came to the OPD of Madinah Teaching Hospital Faisalabad with a chief complaint of suprapubic pain, penile pain, and hard mass on the left side of the scrotum and anterior perineum. The patient had a significant history of undergoing debridement for Meleney's and Fournier's gangrene, along with suprapubic cystostomy done about 1.5 years back. Physical examination revealed a solid mass with dimensions $6 \times 7 \mathrm{~cm}$ on the left side of the scrotum and anterior perineum (left periurethral area). Open surgery was performed. A huge stone, $6 x 6 \mathrm{~cm}$, was removed. Diverticulae were excised, and urethroplasty was performed. A catheter was removed on the 21 st postoperative with a satisfactory urinary stream.

\section{INTRODUCTION}

The incidence of urethral calculus is $<1 \%$ and is more common in males ${ }^{[1]}$. The most common predisposing factors for urethral calculus are stricture urethra, urethral diverticulum, recurrent urinary tract infection, foreign bodies within the urinary tract, and previous surgery on the lower urinary tract ${ }^{[2]}$. Most of the urethral calculi are formed in the kidney or bladder ${ }^{[3,4]}$. Most of these caculi are struvite, calcium phosphate, or calcium carbonate stones ${ }^{[3]}$. Urethral calculus cause dysuria, suprapubic pain, weak urinary stream, urinary tract irritation, acute urinary retention and urethra-cutaneous fistula. Giant urethral calculus is a very rare disease and may be associated with urethral stricture or diverticulum. Open surgery is a conventional approach associated with the risk of urinary tract infections, stenosis and urinary fistulae.

\section{CASE}

A 75-year-old male presented to OPD with suprapubic pain, penile pain and feeling of hard mass in perineum posterior the left side of scrotum. These symptoms had started 2 years back and had increased in intensity in the last 15 days. The patient also had a significant history of undergoing debridement for Meleney's and Fournier's gangrene along with suprapubic cystostomy done 1.5 years back with no history of the passage of urine perurethra since then.
Physical examination showed a significant lower abdominal scar extending over the left side of scrotum with an inserted suprapubic cystostomy. On palpation, a mass of $6 \times 7 \mathrm{~cm}$ was identified posterior to the left side of the scrotum. It was mildly tender. There were no signs of local inflammation. The overlying skin was not separately mobile. Blood parameters, biochemical, coagulation profiles were in a normal range. Urine culture were negative for any growth. X-rays KUB and pelvis, retrograde and antegrade urethrogram before the procedures (Figure: I-II) showed multiple vesicle calculi and a large calculus in the area of the bulbar part of the urethra. On urethroscopy, the distal penile urethra had a pinhole stricture of about $01 \mathrm{~cm}$ (internal urethrotomy was carried out). On cystoscopy, three vesicle calculi of about $3 \times 2 \mathrm{~cm}, 2 \times 2 \mathrm{~cm}$ and $2 \times 1 \mathrm{~cm}$ were found, and cystolitholapaxy was done. Urethral diverticular calculus extraction was conducted by open surgery, and urethroplasty was performed. The dimensions of the stone were $6 \times 6 \mathrm{~cm}$ (Figure-III). A $16 \mathrm{Fr}$ foley catheter was introduced per-urethrally, which was removed on $21 \mathrm{st}$ day with satisfactory stream (Figure: IV-V).

\section{DISCUSSION}

Giant urethral calcululi are rare. Prevalence is high in developing countries ${ }^{[1]}$. Males are more commonly affected, although few cases have been reported in females and children ${ }^{[2]}$. Predisposition factors are diverticulum of urethra, stricture, meatal stenosis with or without hypospadias. Mostly these calculi are struvite ${ }^{[3]}$.

Ramzan A, Akram A, Suaiba S, Jamshed F, Khalid N.Giant urethral stone with diverticulum; a case report. Journal of University Medical \& Dental College. 2021;12(4):290-292. 
Ramzan A, Akram A, Suaiba S, et al.,

Primary calculi are associated with abnormalities like urethral stricture, a foreign body within the urethra or urethral diverticulae. Stones in the urethra with prolonged stay may lead to local necrosis and fibrosis, which is a probable etiology of diverticulum formation ${ }^{[3]}$. Secondary calculi have originated in the kidney or bladder. Primary calculi rarely cause acute symptoms, while secondary calculi can cause dysuria, urine retention, straining during voiding, or even sepsis. The most common treatment modality of giant calculus in the urethral diverticulum is open surgery ${ }^{[4,5,6,7,8]}$. However, with advancements in technology, endoscopic treatment techniques are becoming more popular. Urethral lithotripsy has been successfully performed with a success rate of almost $100 \%{ }^{[9,10]}$.

For emptied urethral diverticulum, diverticulum resection and urethroplasty is usually performed. After endoscopic calculus removal, several conservative treatments like urethral massage and antibiotics for a longer period have to be advised to the patients ${ }^{[9,10]}$. In our case, the urethral diverticulum involved bulbous urethra. After removing calculus, the diverticulum was excised, and urethroplasty was performed with a satisfactory outcome.

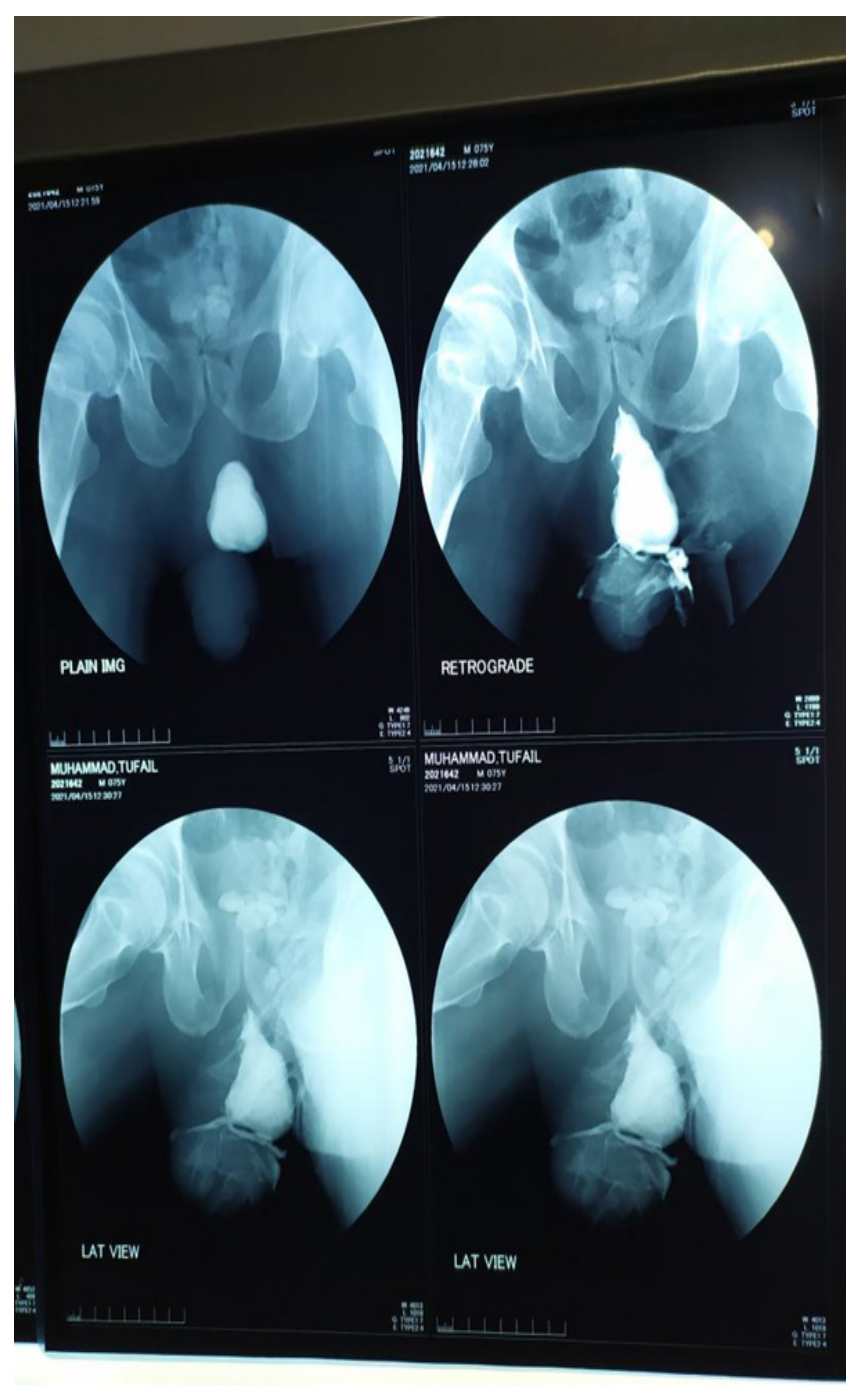

Figure-I: Plain and retrograde urethrogram

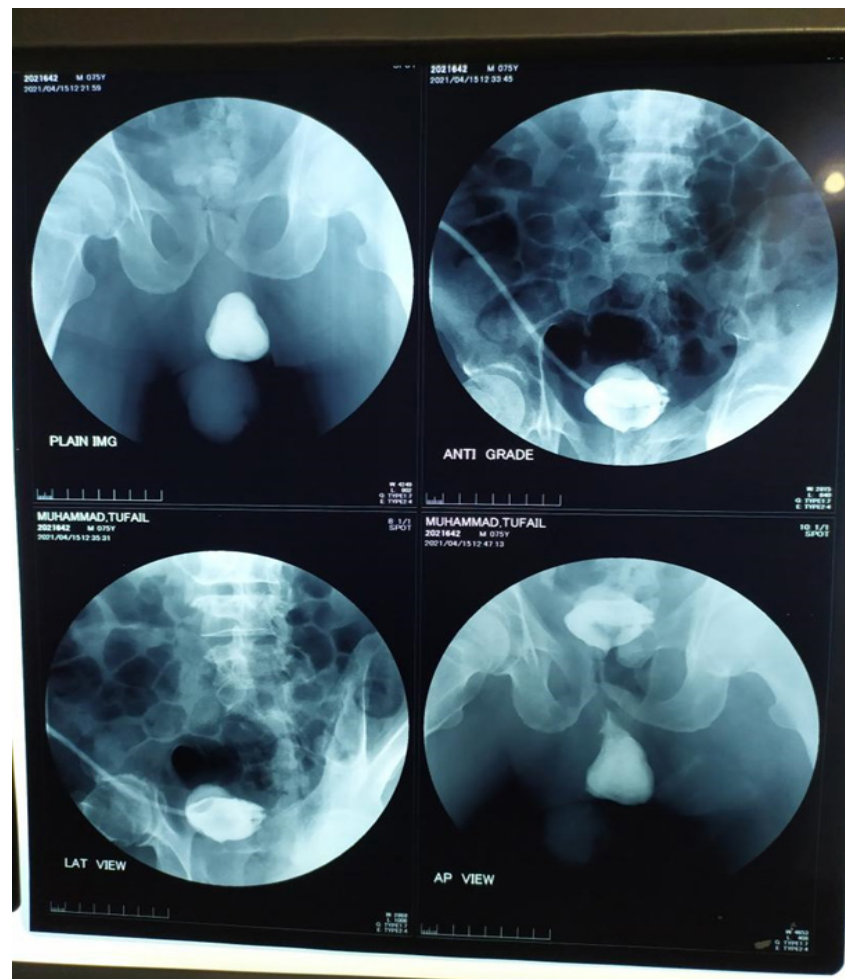

Figure-II: Antegrade urethrogramme.

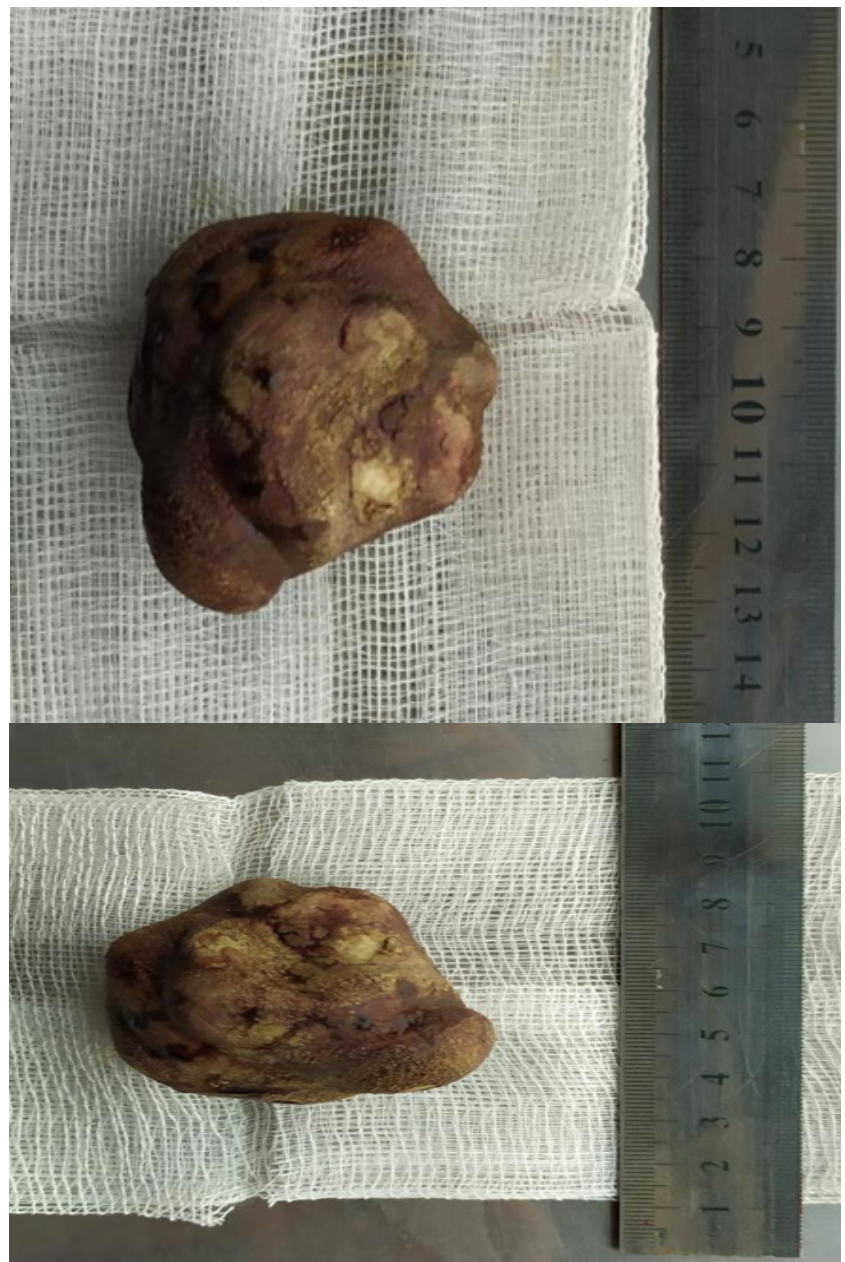

Figure-III: Retrieved stone. 


\section{Giant urethral stone}

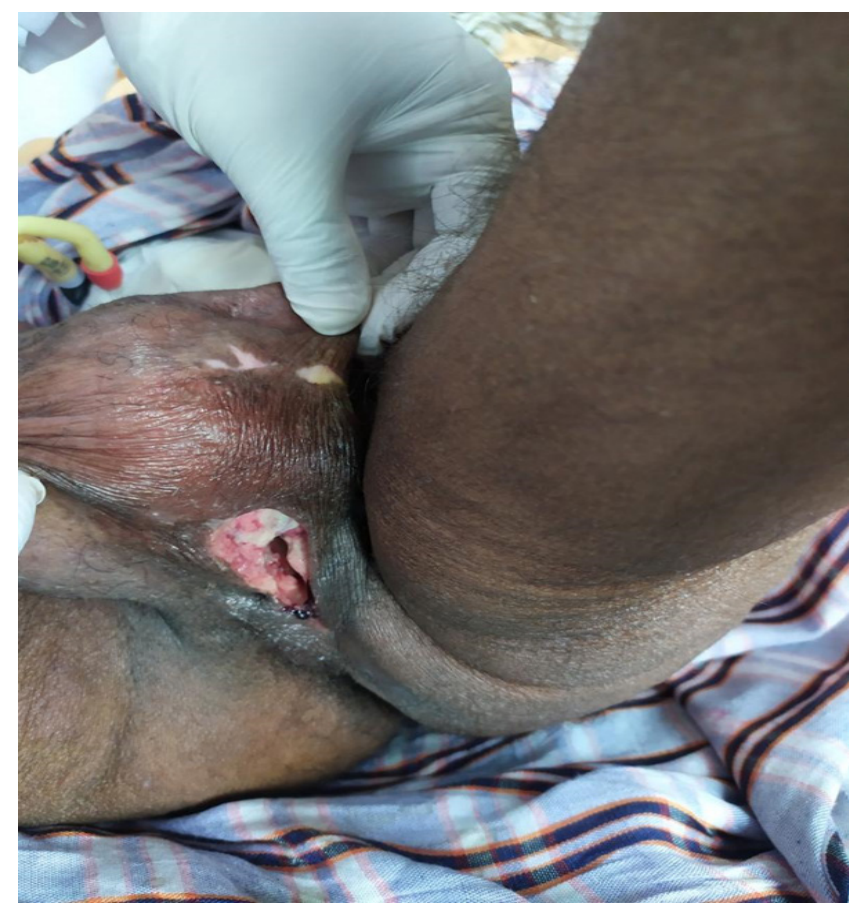

Figure-IV: Urethral diverticulum after stone removal.

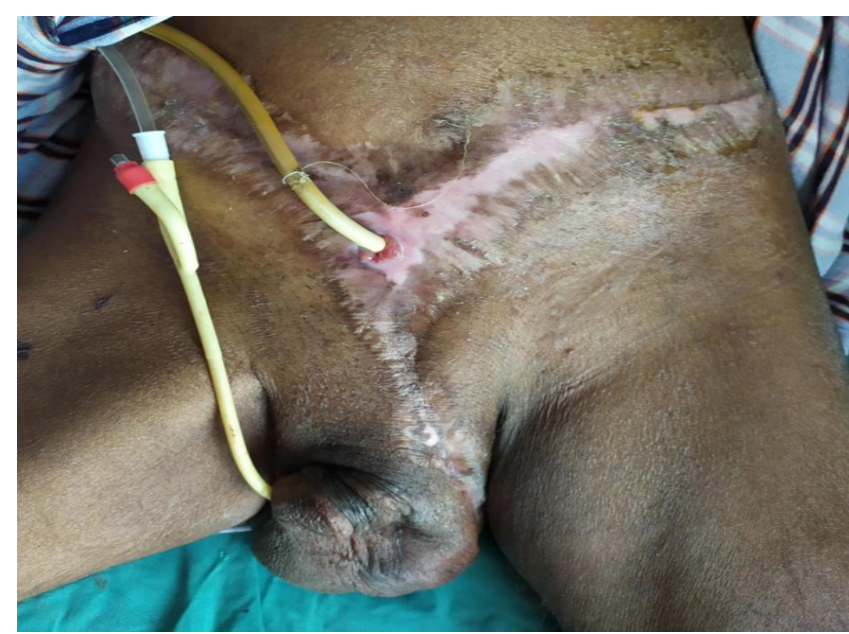

Figure-V: Excision of diverticulum and urethroplasty over foly catheter.

\section{CONCLUSION}

Large urethral calculus in the diverticulum is rare. Accurate diagnosis and treatment can prevent long-term complications.

Permission: Informed written consent from patient was taken for pic and case reporting.

ACKNOWLEDGEMENT: None.

CONFLICT OF INTEREST: All authors declare no conflict of interest.

GRANT SUPPORT \& FINANCIAL DISCLOSURE:

None.

\section{REFERENCES:}

1. Rimon U, Hertz M, Jonas P. Diverticula of the male urethra: a review of 61 cases. Urologic Radiology.
1992;14(1):49-55

2. Mohan V, Gupta SK, Cherian J, Tripathi VN, Sharma BB. Urethral diverticulum in male subjects: report of 5 cases. The Journal of Urology. 1980;123(4):592-594.

3. Marya SK, Kumar S, Singh S. Acquired male urethral diverticulum. The Journal of Urology. 1977;118(5):765766.

4. Allen D, Mishra V, Pepper W, Shah S, Motiwala H. A single-center experience of symptomatic male urethral diverticula. Urology. 2007;70(4):650-653.

5. Ockrim JL, Allen DJ, Shah PJ, Greenwell TJ. A tertiary experience of urethral diverticulectomy: diagnosis, imaging and surgical outcomes. BJU International. 2009;103(11):1550-1554.

6. Alphs HH, Meeks JJ, Casey JT, Gonzalez CM. Surgical reconstruction of the male urethral diverticulum. Urology. 2010;76(2):471-475.

7. Labanaris AP, Zugor V, Witt JH, Nützel R, Kühn R. Urethral diverticulum with massive lithiasis presenting as a scrotal mass. Urologia Internationalis. 2011;87(4):481-483.

8. De Filippo RE, Kurzrock EA, Stein JP, Skinner DG. A giant urethral diverticulum presenting as a scrotal mass in an adult male. British Journal of Urology. 1999;83(4):522-523.

9. Demir O, Kefi A, Cahangirov A, Cihan A, Obuz F, Esen AA, et al. The giant calculus within the prostatic urethra. Urological Research. 2011;39(4):319-321.

10. Sun M, Xu W, Guo S, Ma W, Xu H, Sun R. Giant urethral calculus in anterior urethral diverticulum: a case report. BMC urology. 2019;19(1):1-5. Doi:10.1186/s12894019-0498-9

Author's Contribution:

Asad Ramzan: Conception and design of the work.

Adeen Akram: Substantial contributions to the conception and drafting the work.

Suaiba Saif: Editing of manuscript.

Farhan Jamshed: Critical revision of the article.

Nauman Khalid:Revising it critically for important intellectual content.

Submitted for Publication:07-09-2021 Accepted after revision: 20-10-2021 